\title{
Explicit Solutions to the (3+1)-Dimensional Kudryashov-Sinelshchikov Equations in Bubbly Flow Dynamics
}

\author{
Y. B. Chukkol (D), M. N. B. Mohamad, and Mukhiddin Muminov \\ Department of Mathematical Sciences, Universiti Teknologi Malaysia, 81310 Johor Bahru, Johor, Malaysia \\ Correspondence should be addressed to Y. B. Chukkol; bcyusuf2@live.utm.my
}

Received 26 August 2018; Accepted 2 October 2018; Published 1 November 2018

Academic Editor: Mehmet Sezer

Copyright (c) 2018 Y. B. Chukkol et al. This is an open access article distributed under the Creative Commons Attribution License, which permits unrestricted use, distribution, and reproduction in any medium, provided the original work is properly cited.

A modified tanh-coth method with Riccati equation is used to construct several explicit solutions of $(3+1)$-dimensional Kudryashov-Sinelshchikov equations in bubble gas liquid flow. The solutions include solitons and periodic solutions. The method applied can be used in further works to obtain entirely new solutions to many other nonlinear evolution equations.

\section{Introduction}

Many complex phenomena in nature are describe in various scientific and industrial fields and especially in areas of physics such as fluid mechanics [1], optical fibres [2], plasma physics [3], and so on. Nonlinear evolution equations are used to describe these nonlinear phenomena, and that has led to the development of methods to look for exact solutions of nonlinear partial differential equations [4].

Recently, Kudryashov and Sinelshchikov [5] developed a $(3+1)$-dimensional nonlinear evolution equation in a model of wave propagation in bubbly fluid flow. The model equation has gained a lot of attention where BÃacklund transformation and conservation laws [6], bifurcation [7], and densityfluctuation [8] analysis were carried out the important evolution equation.

Many methods have been developed to find the explicit solutions of nonlinear evolution equations; example of such methods are the first integral method [9], Jacobi elliptic function method [10], Hirota bilinear method [11], Wronskian determinant technique [12], F-expansion method [13], Darboux Transformations [14], Backlund transformation method [6], Miura transformation [15], homotopy perturbation method [16], and Adomian decomposition method [17]. Many algebraic methods were proposed so far, such as tanh method which was proposed by Malflie [18]. Fan [19] extended the tanh method and obtained new exact solution that cannot be obtained by using the conventional tanh method. Further extension called tanh-coth was proposed by Wazwaz [4] which provides a wider applicability for solving nonlinear evolution equations. A modification was also proposed by El-Wakil [20] and Soliman [21, 22].

In this paper, we present two equations $(3+1)$-dimensional Kudryashov and Sinelshchikov equation written as

$$
\begin{array}{r}
\left(u_{t}+\alpha u u_{x}+\gamma u_{x x x}\right)_{x}+d u_{y y}+e u_{z z}=0 \\
\left(u_{t}+\alpha u u_{x}-\beta u_{x x}+\gamma u_{x x x}\right)_{x}+d u_{y y}+e u_{z z}=0
\end{array}
$$

where $\alpha, \beta$, and $\gamma$ represent the nonlinearity, dissipation dispersion terms, while $d$ and $e$ stand for transverse variation of wave in $y$ and $z$ directions; we assume all the coefficient to be constant parameters. We shall use modified tanh-coth to obtain many explicit exact solutions for $(3+1)$-dimensional Kudryashov-Sinelshchikov equations. The travelling wave solution of a special case of (1) is given in [7]. Note that when $e=0$, (1) reduces to their two-dimensional counterparts

$$
\begin{array}{r}
\left(u_{t}+\alpha u u_{x}+\gamma u_{x x x}\right)_{x}+d u_{y y}=0, \\
\left(u_{t}+\alpha u u_{x}-\beta u_{x x}+\gamma u_{x x x}\right)_{x}+d u_{y y}=0,
\end{array}
$$

which were widely studied in $[16,17,23,24]$. Furthermore when $d=e=0$, (1) reduces to one-dimensional Kortewegde-Vries equations $(\mathrm{KdV})$ and one-dimensional Kortewegde-Vries-Burgers equations (KdVB). These equations have many applications in fluid dynamics [25], plasma physics 
[26], cold atomic gases [27], and so on. It is well known that the $\mathrm{KdV}$ and $\mathrm{KdVB}$ equations take the form

$$
\begin{array}{r}
u_{t}+\alpha u u_{x}+\gamma u_{x x x}=0, \\
u_{t}+\alpha u u_{x}-\beta u_{x x}+\gamma u_{x x x}=0 .
\end{array}
$$

These two equations have been extensively investigated by many researcher in recent years [28-32].

In the following section, we briefly describe the modified tanh-coth method in three variables and the application of the modified tanh-coth to $(3+1)$-dimensional Kudryashov and Sinelshchikov equations and their explicit solutions will be given in Section 3. Conclusions will follow in Section 4.

\section{Description of the Method}

Let us consider a three-dimensional nonlinear partial differential equation in the form

$$
u_{t}=F\left(u, u_{x}, u_{y}, u_{z}, u_{x x}, u_{y y}, u_{z z}, \ldots\right) \text {. }
$$

Upon using the transformation $u(x, y, z, t)=u(\xi)$, where $\xi=$ $\mu(x+y+z-c t),(4)$ can be reduced to an ordinary differential equation (ODE)

$$
\begin{aligned}
& -c \mu u^{\prime}(\xi) \\
& \quad=F\left(u(\xi), \mu u^{\prime}(\xi), \mu^{2} u^{\prime \prime}(\xi), \mu^{3} u^{\prime \prime \prime}(\xi), \ldots\right),
\end{aligned}
$$

where the independent variable composed of the new variable $\xi=\mu(x+y+z-c t), \mu$ is the wave number, $c$ is wave speed, $x, y, z$ are the spatial coordinates, and $t$ is time. The ODE (5) is then integrated as long as all terms contain derivatives, where integration constants are considered zero. The simplified ODE is then solved via the tanh-coth method [28], which admits the use of finite expansion

$$
u(Y)=\sum_{i=0}^{m} a_{i} Y^{i}+\sum_{i=1}^{m} b_{i} Y^{-i}
$$

with the Riccati equation

$$
Y^{\prime}=P+Q Y+R Y^{2} .
$$

By changing of variable

$$
\begin{aligned}
\frac{d}{d \xi} & =\left(P+Q Y+R Y^{2}\right) \frac{d}{d Y} \\
\frac{d^{2}}{d \xi^{2}} & =\left(P+Q Y+R Y^{2}\right) \\
\cdot( & \left.(Q+2 R Y) \frac{d}{d Y}+\left(P+Q Y+R Y^{2}\right) \frac{d^{2}}{d Y^{2}}\right),
\end{aligned}
$$

where $P, Q$, and $R$ shall be given, while $a_{i}, b_{i}$ are constants to be determined later. The positive integer $m$ can be determined by considering the homogeneous balance [33] between the highest order derivatives and the most nonlinear terms appearing in (5). If $m$ is not an integer, then a transformation formula should be used to overcome the difficulty. Substituting (6) into (5) and make use of (7) and (8) yield an equation in terms of $Y^{i}$. Equating the coefficients of all the power $Y^{\mathrm{i}}$ to zero, we obtain a set of algebraic equations for $P, Q, R, a_{i}, b_{i}, c$, and $\mu$.

For the aforementioned method, expansion (8) reduces to the standard tanh-coth method. In this work, we shall use the following solutions of Riccati equation:

$$
\begin{aligned}
& P=Q=1 \text { and } R=0 \text {, then } Y=\exp (\xi)-1, \\
& P=1 / 2, Q=0, R=-1 / 2 \text {, then } Y=\operatorname{coth} \xi \pm \operatorname{csch} \xi
\end{aligned}
$$

\section{Application of the Method}

In this section, we will apply the method to the $(3+1)$ dimensional Kudryashov-Sinelshchikov equation for both dispersive and dissipative cases.

3.1. The (3+1)-Dimensional Kudryashov-Sinelshchikov Equation with Dispersion. The generalised equation is given as

$$
\left(u_{t}+\alpha u u_{x}+\gamma u_{x x x}\right)_{x}+d u_{y y}+e u_{z z}=0 .
$$

By using the transformation

$$
\begin{aligned}
u(x, y, z, t) & =u(\xi), \\
\xi & =\mu(x+y+z-c t),
\end{aligned}
$$

(9) is reduced to an ODE of the form

$$
\mu\left(-c \mu u^{\prime}+\alpha \mu u u^{\prime}+\gamma \mu^{3} u^{\prime \prime \prime}\right)^{\prime}+d \mu^{2} u^{\prime \prime}+e \mu^{2} u^{\prime \prime}=0 .
$$

Integrating (11) twice and assuming the integration constant to be zero, we obtain

$$
(-c+d+e) u+\frac{\alpha}{2} u^{2}+\gamma \mu^{2} u^{\prime \prime}=0
$$

Balancing terms $u^{2}$ and $u^{\prime \prime}$ in (12), we get $m=2$, which enables us to make the following ansatz:

$$
u(\xi)=a_{0}+a_{1} Y+a_{2} Y^{2}+\frac{b_{1}}{Y}+\frac{b_{2}}{Y^{2}} .
$$

Substituting (13) into (12) and making use of (7) and (8), we obtain a system of equation in terms of $a_{i}(i=0,1,2), b_{i}(i=$ $1,2), \alpha, \gamma, d$, e $c, P, Q, R$, and $\mu$.

$$
\begin{aligned}
& 12 \gamma R^{2} \mu^{2} a_{2}+\alpha a_{2}{ }^{2}=0, \\
& 4 \gamma a_{1} R^{2} \mu^{2}+20 \gamma Q a_{2} R \mu^{2}+2 \alpha a_{1} a_{2}=0, \\
& 8 \gamma a_{2} Q^{2} \mu^{2}+6 \gamma R Q \mu^{2} a_{1}+16 \gamma P R a_{2} \mu^{2}+\alpha a_{1}^{2}+2 d a_{2} \\
& \quad+2 e a_{2}-2 c a_{2}+2 \alpha a_{0} a_{2}=0, \\
& 2 \gamma a_{1} Q^{2} \mu^{2}+12 \gamma P a_{2} Q \mu^{2}+4 \gamma P R a_{1} \mu^{2}+2 d a_{1}+2 e a_{1} \\
& \quad-2 c a_{1}+2 \alpha a_{0} a_{1}+2 \alpha a_{2} b_{1}=0,
\end{aligned}
$$




$$
\begin{aligned}
& 2 d a_{0}+2 e a_{0}-2 c a_{0}+\alpha a_{0}^{2}+2 \alpha a_{1} b_{1}+2 \alpha a_{2} b_{2} \\
& \quad+4 \gamma P^{2} \mu^{2} a_{2}+4 \gamma R^{2} \mu^{2} b_{2}+2 \gamma P Q \mu^{2} a_{1} \\
& \quad+2 \gamma Q R \mu^{2} b_{1}=0, \\
& 2 \gamma b_{1} Q^{2} \mu^{2}+12 \gamma R b_{2} Q \mu^{2}+4 \gamma P R b_{1} \mu^{2}+2 d b_{1}+2 e b_{1} \\
& \quad-2 c b_{1}+2 \alpha a_{0} b_{1}+2 \alpha a_{1} b_{2}=0, \\
& 8 \gamma b_{2} Q^{2} \mu^{2}+6 \gamma P Q \mu^{2} b_{1}+16 \gamma P R b_{2} \mu^{2}+\alpha b_{1}^{2}+2 d b_{2} \\
& \quad+2 e b_{2}-2 c b_{2}+2 \alpha a_{0} b_{2}=0, \\
& 4 \gamma b_{1} P^{2} \mu^{2}+20 \gamma Q b_{2} P \mu^{2}+2 \alpha b_{1} b_{2}=0, \\
& 12 \gamma P^{2} \mu^{2} b_{2}+\alpha b_{2}^{2}=0 .
\end{aligned}
$$

Case I. If $P=1, Q=1, R=0$ are substituted into the system of (14) and solving the equations, we have the following solutions:

$$
\begin{aligned}
& a_{0}=\frac{2(c-d-e)}{\alpha}, \\
& a_{1}=a_{2}=0, \\
& b_{1}=b_{2}=6 a_{0}, \\
& \mu=\frac{\sqrt{-\gamma(c-d-e)}}{\gamma}, \\
& a_{0}=a_{1}=a_{2}=0, \\
& b_{1}=b_{2}=-\frac{12(c-d-e)}{\alpha}, \\
& \mu=\frac{\sqrt{\gamma(c-d-e)}}{\gamma} .
\end{aligned}
$$

Substituting (15a) and (15b) into (13) using $Y=\exp (\xi)-1, \gamma>$ 0 , with $\Omega=x+y+z-c t$, we get the solutions in simple form as

$$
\begin{aligned}
& u_{1}(x, y, z, t) \\
& =\frac{2(c-d-e)}{\alpha} \\
& \left.\quad+\frac{3(c-d-e)}{\alpha \sinh ^{2}((\sqrt{-\gamma(c-d-e)} / \gamma)(\Omega / 2)}\right) \\
& \quad c<d+e \\
& u_{2}(x, y, z, t)=-\frac{3(c-d-e)}{\alpha \sinh ^{2}((\sqrt{\gamma(c-d-e)} / \gamma)(\Omega / 2))}, \\
& c>d+e .
\end{aligned}
$$

Case II. If we set $P=1 / 2, Q=0, R=-1 / 2$ into the system of (14) and solve the equations, we have the following solutions:

$$
\begin{aligned}
& a_{0}=-\frac{c-d-e}{\alpha}, \\
& a_{1}=0 \text {, } \\
& a_{2}=-3 a_{0} \text {, } \\
& b_{1}=b_{2}=0 \text {, } \\
& \mu=\frac{\sqrt{-\gamma(c-d-e)}}{\gamma}, \\
& a_{0}=\frac{3(c-d-e)}{\alpha}, \\
& a_{1}=0 \text {, } \\
& a_{2}=-3 a_{0} \text {, } \\
& b_{1}=b_{2}=0 \text {, } \\
& \mu=\frac{\sqrt{\gamma(c-d-e)}}{\gamma}, \\
& a_{0}=-\frac{c-d-e}{\alpha}, \\
& a_{1}=a_{2}=0 \text {, } \\
& b_{1}=0 \text {, } \\
& b_{2}=-3 a_{0} \text {, } \\
& \mu=\frac{\sqrt{-\gamma(c-d-e)}}{\gamma}, \\
& a_{0}=\frac{3(c-d-e)}{\alpha}, \\
& a_{1}=a_{2}=0 \text {, } \\
& b_{1}=0 \text {, } \\
& b_{2}=-a_{0} \text {, } \\
& \mu=\frac{\sqrt{\gamma(c-d-e)}}{\gamma}, \\
& a_{0}=\frac{c-d-e}{2 \alpha}, \\
& a_{1}=b_{1}=0 \text {, } \\
& a_{2}=b_{2}=\frac{3(c-d-e)}{4 \alpha} \text {, } \\
& \mu=\frac{\sqrt{-\gamma(c-d-e)}}{2 \gamma}, \\
& a_{0}=\frac{3(c-d-e)}{2 \alpha}, \\
& a_{1}=b_{1}=0 \text {, }
\end{aligned}
$$




$$
\begin{gathered}
a_{2}=b_{2}=-\frac{3(c-d-e)}{4 \alpha}, \\
\mu=\frac{\sqrt{\gamma(c-d-e)}}{2 \gamma} .
\end{gathered}
$$$$
=\frac{2(c-d-e)}{\alpha}
$$$$
-\frac{3(c-d-e)}{\alpha \cosh ^{2}((\sqrt{-\gamma(c-d-e)} / \gamma)(\Omega / 2))},
$$

Substituting $Y=\operatorname{coth} \xi \pm \operatorname{csch} \xi, \gamma>0, \Omega=x+y+z-c t$ into (13), making use of (18a), we obtain a soliton solution

$$
u_{3}(x, y, z, t)
$$

and periodic solutions

$$
\begin{aligned}
& u_{4}(x, y, z, t)=\frac{2(c-d-e)}{\alpha}-\frac{3(c-d-e)}{\alpha \cos ^{2}((\sqrt{\gamma(c-d-e)} / \gamma)(\Omega / 2))}, \quad c>d+e \\
& u_{5}(x, y, z, t)=\frac{2(c-d-e)}{\alpha}-\frac{6(c-d-e)}{\alpha(\cos ((\sqrt{\gamma(c-d-e)} / \gamma)(\Omega / 2))+\sin ((\sqrt{\gamma(c-d-e)} / \gamma)(\Omega / 2)))^{2}},
\end{aligned}
$$

where $c>d+e$. Using (18b), we have a soliton solution, written in simple form as

$$
\begin{array}{r}
u_{6}(x, y, z, t)=\frac{3(c-d-e)}{\alpha \cosh ^{2}((\sqrt{\gamma(c-d-e)} / \gamma)(\Omega / 2))}, \\
c>d+e
\end{array}
$$

and periodic solutions

$$
\begin{aligned}
& u_{7}(x, y, z, t)=\frac{3(c-d-e)}{\alpha \cos ^{2}((\sqrt{-\gamma(c-d-e)} / \gamma)(\Omega / 2))}, \quad c<d+e \\
& u_{8}(x, y, z, t)=\frac{6(c-d-e)}{\alpha(\cos ((\sqrt{-\gamma(c-d-e)} / \gamma)(\Omega / 2))+\sin ((\sqrt{-\gamma(c-d-e)} / \gamma)(\Omega / 2)))^{2}}, \quad c<d+e .
\end{aligned}
$$

Equation (18c) gives a soliton solution

$$
\begin{aligned}
& u_{9}(x, y, z, t) \\
& =\frac{2(c-d-e)}{\alpha} \\
& u_{10}(x, y, z, t)=\frac{2(c-d-e)}{\alpha}-\frac{c<d+e}{\alpha \sin ^{2}((\sqrt{\gamma(c-d-e)} / \gamma)(\Omega / 2))}, \quad c>d+e \\
& u_{11}(x, y, z, t)=\frac{2(c-d-e)}{\alpha}-\frac{3(c-d-e)}{\alpha(\cos ((\sqrt{\gamma(c-d-e)} / \gamma)(\Omega / 2))-\sin ((\sqrt{\gamma(c-d-e) / \gamma})(\Omega / 2)))^{2}},
\end{aligned}
$$$$
+\frac{3(c-d-e)}{\alpha \sinh ^{2}((\sqrt{-\gamma(c-d-e)} / \gamma)(\Omega / 2))},
$$

where $c>d+e$. Equation (18d) gives a soliton solution

$$
=-\frac{3(c-d-e)}{\alpha \sinh ^{2}((\sqrt{\gamma(c-d-e)} / \gamma)(\Omega / 2))},
$$$$
c>d+e
$$$$
u_{12}(x, y, z, t)
$$ 
and periodic solutions

$$
\begin{aligned}
& u_{13}(x, y, z, t)=\frac{3(c-d-e)}{\alpha \sin ^{2}((\sqrt{-\gamma(c-d-e)} / \gamma)(\Omega / 2))}, \quad c<d+e \\
& u_{14}(x, y, z, t)=\frac{6(c-d-e)}{\alpha(\cos ((\sqrt{-\gamma(c-d-e)} / \gamma)(\Omega / 2))+\sin ((\sqrt{-\gamma(c-d-e)} / \gamma)(\Omega / 2)))^{2}}, \quad c<d+e .
\end{aligned}
$$

Equation (18e) gives soliton solutions

$$
\begin{aligned}
& u_{15}(x, y, z, t) \\
& =\frac{2(c-d-e)}{\alpha} \\
& \quad+\frac{3(c-d-e)}{\alpha \sinh ^{2}((\sqrt{-\gamma(c-d-e)} / \gamma)(\Omega / 2))}, \\
& \quad c<d+e \\
& u_{16}(x, y, z, t) \\
& =\frac{2(c-d-e)}{\alpha} \quad c<d+e
\end{aligned}
$$

and periodic solutions

$$
\begin{aligned}
& u_{17}(x, y, z, t) \\
& =\frac{2(c-d-e)}{\alpha}, \\
& \quad-\frac{3(c-d-e)}{\alpha \sin ^{2}((\sqrt{\gamma(c-d-e)} / \gamma)(\Omega / 2))}, \\
& u_{18}(x, y, z, t) \\
& =\frac{2(c-d-e)}{\alpha} \quad c>d+e \\
& \quad-\frac{3(c-d-e)}{\alpha \cos ^{2}((\sqrt{\gamma(c-d-e)} / \gamma)(\Omega / 2))},
\end{aligned}
$$

$$
\left(u_{t}+\alpha u u_{x}-\beta u_{x x}+\gamma u_{x x x}\right)_{x}+d u_{y y}+e u_{z z}=0 .
$$

By using the transformation

$$
\begin{aligned}
u(x, y, z, t) & =u(\xi), \\
\xi & =\mu(x+y+z-c t),
\end{aligned}
$$

(39) is reduced to an ODE of the form

$$
\begin{aligned}
& \mu\left(-c \mu u^{\prime}+\alpha \mu u u^{\prime}-\beta \mu^{2} u^{\prime \prime}+\gamma \mu^{3} u^{\prime \prime \prime}\right)^{\prime}+d \mu^{2} u^{\prime \prime} \\
& +e \mu^{2} u^{\prime \prime}=0 .
\end{aligned}
$$

$$
c<d+e .
$$

and periodic solutions

The graphical representation of the solitary wave profile to the solution $u_{3}(x, y, z, t)$ of (9) at $t=0$ is given in Figure 1,

3.2. The (3+1)-Dimensional Kudryashov-Sinelshchikov Equation with Dispersion and Dissipation. The equation is given as

Integrating (41) twice and assuming the integration constant to be zero, we obtain

$$
(-c+d+e) u+\frac{\alpha}{2} u^{2}-\beta \mu u^{\prime}+\gamma \mu^{2} u^{\prime \prime}=0 .
$$

$$
\begin{aligned}
& u_{19}(x, y, z, t) \\
& =-\frac{3(c-d-e)}{\alpha \sinh ^{2}((\sqrt{\gamma(c-d-e)} / \gamma)(\Omega / 2))},
\end{aligned}
$$

Balancing $u^{2}$ and $u^{\prime \prime}$ in (42), we get $m=2$, which enables us to make the following ansatz:

$$
u(\xi)=a_{0}+a_{1} Y+a_{2} Y^{2}+\frac{b_{1}}{Y}+\frac{b_{2}}{Y^{2}} .
$$




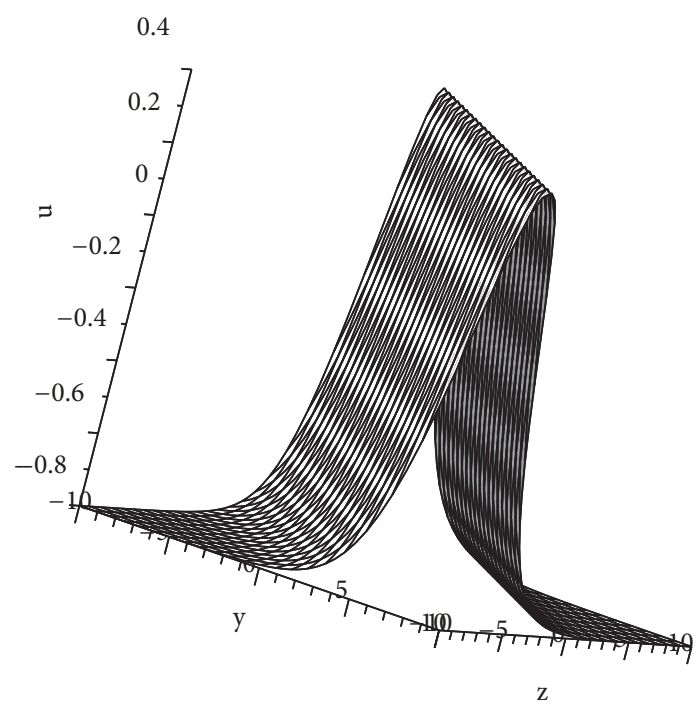

FIGURE 1: Solitary wave profile of $u_{3}, \alpha=1, d=0.5, e=0.5, c=0.6$, $x=0$, and $t=0$.

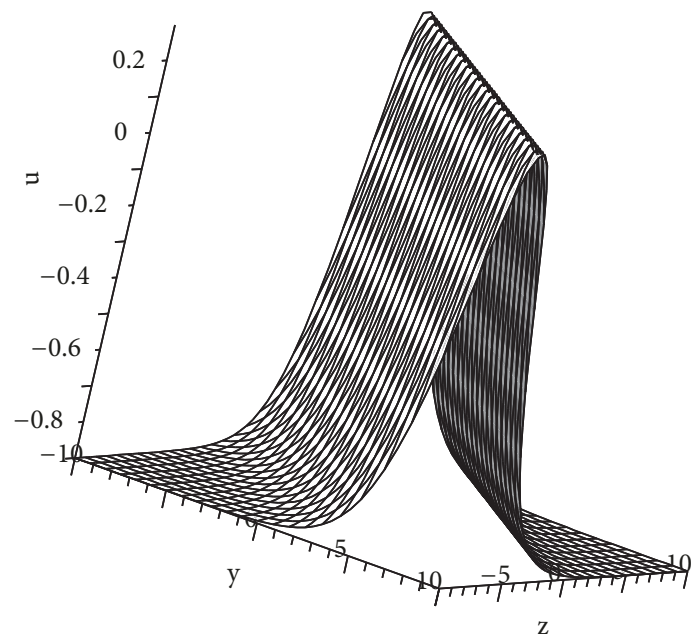

FIGURE 2: Solitary wave profile of $u_{3}, \alpha=1, d=0.5, e=0.5, c=0.6$, $x=0$, and $t=0.5$.

Substituting (43) into (42) and making use of (7) and (8), we obtained a system of equations in $a_{i}(i=0,1,2), b_{i}(i=$ $1,2), c, P, Q, R, \alpha, \beta, \gamma$, and $\mu$.

$$
\begin{aligned}
& 12 \gamma R^{2} \mu^{2} a_{2}+\alpha a_{2}{ }^{2}=0, \\
& 4 \gamma a_{1} R^{2} \mu^{2}+20 \gamma Q a_{2} R \mu^{2}-4 \beta a_{2} R \mu+2 \alpha a_{1} a_{2}=0, \\
& 8 \gamma a_{2} Q^{2} \mu^{2}+6 \gamma R Q \mu^{2} a_{1}-4 \beta a_{2} Q \mu+16 \gamma P R a_{2} \mu^{2} \\
& \quad-2 \beta R \mu a_{1}+\alpha a_{1}^{2}+2 d a_{2}+2 e a_{2}-2 c a_{2} \\
& \quad+2 \alpha a_{0} a_{2}=0, \\
& 2 \gamma a_{1} Q^{2} \mu^{2}+12 \gamma P a_{2} Q \mu^{2}-2 \beta a_{1} Q \mu+4 \gamma A R a_{1} \mu^{2} \\
& \quad-4 \beta P a_{2} \mu+2 d a_{1}+2 e a_{1}-2 c a_{1}+2 \alpha a_{0} a_{1} \\
& \quad+2 \alpha a_{2} b_{1}=0,
\end{aligned}
$$

$$
\begin{aligned}
& 2 d a_{0}+2 e a_{0}-2 w a_{0}+\alpha a_{0}^{2}+2 \alpha a_{1} b_{1}+2 \alpha a_{2} b_{2} \\
& +4 \gamma P^{2} \mu^{2} a_{2}+4 \gamma R^{2} \mu^{2} b_{2}-2 \beta P \mu a_{1}+2 \beta R \mu b_{1} \\
& +2 \gamma P Q \mu^{2} a_{1}+2 \gamma Q R \mu^{2} b_{1}=0, \\
& 2 \gamma b_{1} Q^{2} \mu^{2}+12 \gamma R b_{2} Q \mu^{2}+2 \beta b_{1} Q \mu+4 \gamma P R b_{1} \mu^{2} \\
& +4 \beta R b_{2} \mu+2 d b_{1}+2 e b_{1}-2 c b_{1}+2 \alpha a_{0} b_{1} \\
& +2 \alpha a_{1} b_{2}=0,
\end{aligned}
$$

$$
\begin{aligned}
& 8 \gamma b_{2} Q^{2} \mu^{2}+6 \gamma P Q \mu^{2} b_{1}+4 \beta b_{2} Q \mu+16 \gamma P Q b_{2} \mu^{2} \\
& \quad+2 \beta P \mu b_{1}+\alpha b_{1}^{2}+2 d b_{2}+2 e b_{2}-2 c b_{2} \\
& \quad+2 \alpha a_{0} b_{2}=0
\end{aligned}
$$$$
4 \gamma b_{1} P^{2} \mu^{2}+20 \gamma Q b_{2} P \mu^{2}+4 \beta b_{2} P \mu+2 \alpha b_{1} b_{2}=0 \text {, }
$$$$
12 \gamma P^{2} \mu^{2} b_{2}+\alpha b_{2}^{2}=0
$$

Case I. If $P=1, Q=1, R=0$ are used in the system (44) and solving the equations, we have obtained the following solutions:

$$
\begin{aligned}
& a_{0}=a_{1}=a_{2}=0, \\
& b_{1}=-\frac{24 \beta^{2}}{25 \gamma \alpha}, \\
& b_{2}=\frac{1}{2} b_{1}, \\
& \mu=\frac{\beta}{5 \gamma}, \\
& c=\frac{6 \beta^{2}+25 \gamma d+25 \gamma e}{25 \gamma}, \\
& a_{0}=b_{2}=-\frac{12 \beta^{2}}{25 \gamma \alpha}, \\
& a_{1}=a_{2}=0, \\
& b_{1}=2 b_{2}, \\
& \mu=\frac{\beta}{5 \gamma}, \\
& c=\frac{25 \gamma d+25 \gamma e-6 \beta^{2}}{25 \gamma} .
\end{aligned}
$$

Substituting (45a) and (45b) into (43) and using $Y=\exp (\xi)-$ 1 , we get

$$
\begin{aligned}
& u_{1}(x, y, z, t)=\frac{12 \beta^{2}}{25 \gamma \alpha}\left[1-(\exp (-\xi)-1)^{-2}\right], \\
& \xi=\frac{\beta}{5 \gamma}\left(x+y+z-\frac{25 \gamma d+25 \gamma e+6 \beta^{2}}{25 \gamma} t\right),
\end{aligned}
$$


and

$$
\begin{aligned}
& u_{2}(x, y, z, t)=-\frac{12 \beta^{2}}{25 \gamma \alpha}(\exp (-\xi)-1)^{-2}, \\
& \xi=\frac{\beta}{5 \gamma}\left(x+y+z-\frac{25 \gamma d+25 \gamma e-6 \beta^{2}}{25 \gamma} t\right)
\end{aligned}
$$

Case II. If we set $P=1 / 2, Q=0$, and $R=-1 / 2$ into system (44) and solve the equation, we have

$$
\begin{aligned}
& a_{0}=a_{2}=-\frac{3 \beta^{2}}{25 \gamma \alpha}, \\
& a_{1}=2 a_{0}=\text {, } \\
& b_{1}=b_{2}=0 \text {, } \\
& \mu=\frac{\beta}{5 \gamma} \text {, } \\
& c=\frac{25 \gamma d+25 \gamma e-6 \beta^{2}}{25 \gamma} \text {, } \\
& a_{0}=\frac{9 \beta^{2}}{25 \gamma \alpha} \text {, } \\
& a_{1}=\frac{-2 a_{0}}{3} \text {, } \\
& a_{2}=-\frac{a_{0}}{3}, \\
& b_{1}=b_{2}=0 \text {, } \\
& \mu=\frac{\beta}{5 \gamma} \text {, } \\
& c=\frac{25 \gamma d+25 \gamma e+6 \beta^{2}}{25 \gamma} \text {, } \\
& a_{0}=b_{2}=-\frac{3 \beta^{2}}{25 \gamma \alpha}, \\
& a_{1}=a_{2}=0 \text {, } \\
& b_{1}=2 b_{2} \text {, } \\
& \mu=\frac{\beta}{5 \gamma} \text {, } \\
& c=\frac{25 \gamma d+25 \gamma e-6 \beta^{2}}{25 \gamma}, \\
& a_{0}=\frac{9 \beta^{2}}{25 \gamma \alpha}, \\
& a_{1}=a_{2}=0 \text {, } \\
& b_{1}=-\frac{2 a_{0}}{3} \text {, }
\end{aligned}
$$

$$
\begin{aligned}
& b_{2}=-\frac{a_{0}}{3}, \\
& \mu=\frac{\beta}{5 \gamma}, \\
& c=\frac{25 \gamma d+25 \gamma e+6 \beta^{2}}{25 \gamma},
\end{aligned}
$$

It can be shown that if $Y=\operatorname{coth} \xi \pm \operatorname{csch} \xi$, the set of values in (48a), (48c), and (48e) give the same solution as

$$
\begin{aligned}
u_{3,4}(x, y, z, t) & \\
= & -\frac{3 \beta^{2}}{25 \gamma \alpha}-\frac{6 \beta^{2}}{25 \gamma \alpha}(\operatorname{coth} \xi \pm \operatorname{csch} \xi) \\
& -\frac{3 \beta^{2}}{25 \gamma \alpha}(\operatorname{coth} \xi \pm \operatorname{csch} \xi)^{2} \\
= & -\frac{12 \beta^{2}}{25 \gamma \alpha}(\exp (-\xi) \mp 1)^{-2}, \\
\xi= & \frac{\beta}{5 \gamma}\left(x+y+z-\frac{25 \gamma d+25 \gamma e-6 \beta^{2}}{25 \gamma} t\right) .
\end{aligned}
$$

The set values in (48b), (48d), and (48f) give exactly the same solution as

$$
\begin{aligned}
& u_{5,6}(x, y, z, t) \\
& \quad=\frac{9 \beta^{2}}{25 \gamma \alpha}-\frac{6 \beta^{2}}{25 \gamma \alpha}(\operatorname{coth} \xi \pm \operatorname{csch} \xi)
\end{aligned}
$$




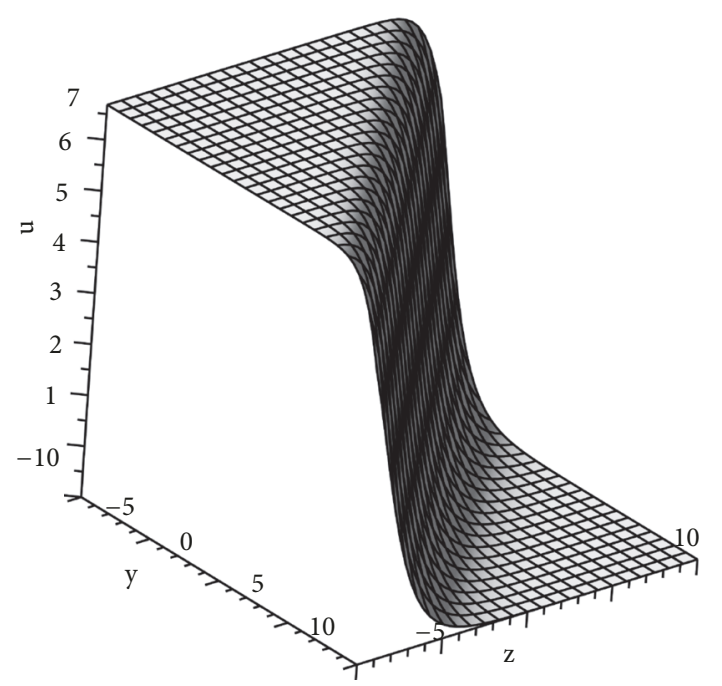

FIGURE 3: Kink wave profile of $u_{5,6}, \alpha=1, \gamma=1, d=0.5, e=0.5$, $\beta=4, x=0$, and $t=0$.

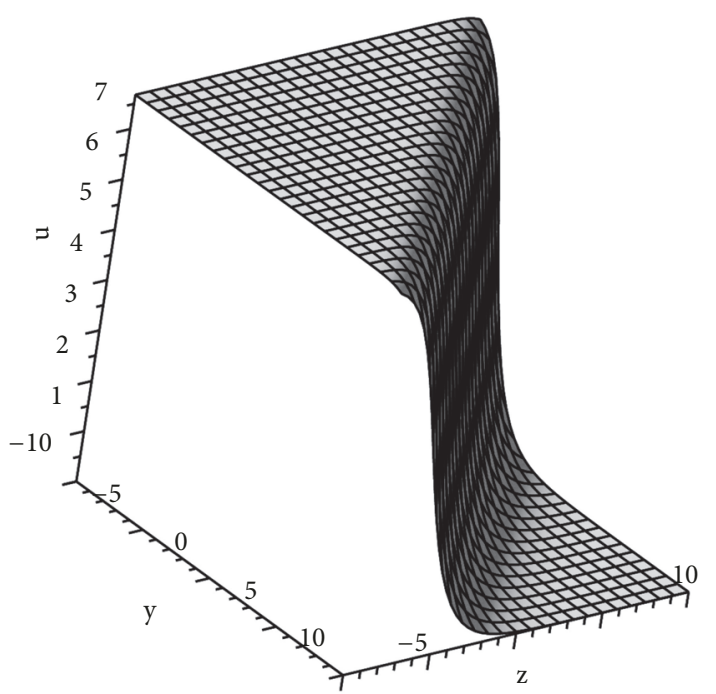

FIGURE 4: Kink wave profile of $u_{5,6}, \alpha=1, \gamma=1, d=0.5, e=0.5$, $\beta=4, x=0$, and $t=0.5$.

$$
\begin{aligned}
& -\frac{3 \beta^{2}}{25 \gamma \alpha}(\operatorname{coth} \xi \pm \operatorname{csch} \xi)^{2} \\
= & \frac{12 \beta^{2}}{25 \gamma \alpha}\left[1-(\exp (-\xi) \mp 1)^{-2}\right], \\
\xi= & \frac{\beta}{5 \gamma}\left(x+y+z-\frac{25 \gamma d+25 \gamma e+6 \beta^{2}}{25 \gamma} t\right) .
\end{aligned}
$$

We use $u_{5,6}(x, y, z, t)$ to represent the solution to (39) graphically; Figure 3 gives a kink wave profile at $t=0$, while at $t=0.5$ it is given in Figure 4 .

\section{Conclusion}

In this paper, the modified tanh-coth method has been applied to nonlinear evolution equations. The explicit travelling wave solutions of the generalised $(3+1)$-dimensional Kudryashov-Sinelshchikov equation are derived. Some of the solutions obtained are in good agreement with the known exact solutions [7] using specific parameter values. The results show that the modified tanh-coth method is an efficient approach for the seeking solutions of such type of nonlinear evolution equations.

\section{Data Availability}

The data used to support the findings of this study are included within the article.

\section{Conflicts of Interest}

The authors declare that there are no conflicts of interest regarding the publication of this paper.

\section{References}

[1] D. L. Albernaz and F. R. Cunha, "Unsteady motion of a spherical bubble in a complex fluid: mathematical modelling and simulation," Applied Mathematical Modelling: Simulation and Computation for Engineering and Environmental Systems, vol. 37, no. 20-21, pp. 8972-8984, 2013.

[2] F. Najafi, J. Mower, N. Harris C et al., "On-chip detection of non-classical light by scalable integration of single-photon detectors," Nature communications, vol. 6, p. 5873, 2015.

[3] K. Ostrikov, E. C. Neyts, and M. Meyyappan, "Plasma nanoscience: from nano-solids in plasmas to nano-plasmas in solids," Advances in Physics, vol. 62, no. 2, pp. 113-224, 2013.

[4] A. Wazwaz, Partial Differential Equations and Solitary Waves Theory, Springer, Berlin, Germany, 2010.

[5] N. Kudryashov and D. I. Sinelshchikov, "Equation for the three-dimensional nonlinear waves in liquid with gas bubbles," Physica Scripta, vol. 85, p. 025402, 2012.

[6] J.-M. Tu, S.-F. Tian, M.-J. Xu, X.-Q. Song, and T.-T. Zhang, "Bäcklund transformation, infinite conservation laws and periodic wave solutions of a generalized (3+1)-dimensional nonlinear wave in liquid with gas bubbles," Nonlinear Dynamics, vol. 83, no. 3, pp. 1199-1215, 2016.

[7] Y. Feng, W. Shan, W. Sun, H. Zhong, and B. Tian, "Bifurcation analysis and solutions of a three-dimensional KudryashovSinelshchikov equation in the bubbly liquid," Communications in Nonlinear Science and Numerical Simulation, vol. 19, no. 4, pp. 880-886, 2014.

[8] X. Gao Y, "Density-fluctuation symbolic computation on the $(3+1)$-dimensional variable-coefficient Kudryashov-Sinelshchikov equation for a bubbly liquid with experimental support," Modern Physics Letters B, vol. 30, p. 1650217, 2016.

[9] M. Mirzazadeh and M. Eslami, "Exact solutions of the Kudryashov-Sinelshchikov equation and nonlinear telegraph equation via the first integral method," Nonlinear Analysis: Modelling and Control, vol. 17, no. 4, pp. 481-488, 2012.

[10] S. Singh and S. Ray, "Exact solutions for the Wick-type stochastic Kersten-Krasil'shchik coupled KdV-mKdV equations," The European Physical Journal Plus, vol. 132, 480 pages, 2017. 
[11] W. Rui and X. Qi, "Bilinear approach to quasi-periodic wave solutions of the Kersten-Krasil'shchik coupled KdV-mKdV system," Boundary Value Problems, p. 130, 2016.

[12] J. Wu, "N-soliton solution, generalized double Wronskian determinant solution and rational solution for a $(2+1)$-dimensional nonlinear evolution equation," Physics Letters A, vol. 373, pp. 83-88, 2008.

[13] A. Ebaid and E. H. Aly, "Exact solutions for the transformed reduced Ostrovsky equation via the $F$-expansion method in terms of Weierstrass-elliptic and Jacobian-elliptic functions," Wave Motion, vol. 49, no. 2, pp. 296-308, 2012.

[14] Z. Ru-Guang and C. Jie, "Two Hierarchies of New DifferentialDifference Equations Related to the Darboux Transformations of the Kaup-Newell Hierarchy," Communications in Theoretical Physics, vol. 63, no. 1, 2015.

[15] Z. Jun-Yi and G. Xian-Guo, "Miura transformation for the TD hierarchy," Chinese Physics Letters, vol. 23, 1 page, 2006.

[16] A. Molabahrami, F. Khani, and S. Hamedi-Nezhad, "Soliton solutions of the two-dimensional KdV-Burgers equation by homotopy perturbation method," Physics Letters A, vol. 370, no. 5-6, pp. 433-436, 2007.

[17] D. Kaya, "An application of the decompositionmethod for the two-dimensional KdV-Burgers equation," Computers \& Mathematics with Applications, vol. 48, pp. 1659-1665, 2004.

[18] W. Malfliet and W. Hereman, "The tanh method. I. Exact solutions of nonlinear evolution and wave equations," Physica Scripta, vol. 54, no. 6, pp. 563-568, 1996.

[19] E. Fan, J. Zhang, and B. Y. Hon, "A new complex line soliton for the two-dimensional KdV-Burgers equation," Physics Letters A, vol. 291, no. 6, pp. 376-380, 2001.

[20] S. A. El-Wakil and M. A. Abdou, "New exact travelling wave solutions using modified extended tanh-function method," Chaos, Solitons \& Fractals, vol. 31, pp. 1256-1264, 2007.

[21] A. A. Soliman, "The modified extended tanh-function method for solving Burgers-type equations," Physica A: Statistical Mechanics and its Applications, vol. 361, no. 2, pp. 394-404, 2006.

[22] M. Abdou and A. A. Soliman, "Modified extended tanh-function method and its application on nonlinear physical equations," Physics Letters A, vol. 353, pp. 487-492, 2006.

[23] M. J. Ablowitz, A. Demirci, and Y.-P. Ma, "Dispersive shock waves in the Kadomtsev-Petviashvili and two dimensional Benjamin-Ono equations," Physica D: Nonlinear Phenomena, vol. 333, pp. 84-98, 2016.

[24] T. Grava, C. Klein, and G. Pitton, "Numerical study of the Kadomtsev-Petviashvili equation and dispersive shock waves," Proceedings of the Royal Society of London. Series A, vol. 474, no. 2210, p. 20170458, 2018.

[25] N. A. Kudryashov and D. I. Sinelshchikov, "Nonlinear waves in bubbly liquids with consideration for viscosity and heat transfer," Physics Letters A, vol. 374, pp. 2011-2016, 2010.

[26] I. Kourakis, S. Sultana, and F. Verheest, "Note on the singleshock solutions of the Korteweg-de Vries-Burgers equation," Astrophysics and Space Science, vol. 338, no. 2, pp. 245-249, 2012.

[27] M. Kulkarni and A. Abanov G, "Hydrodynamics and universality in cold atomic gases," Physical Review A, vol. 86, p. 033614, 2012.

[28] L. Wazzan, "A modified tanh-coth method for solving the KDV and the KDV-BUrgers' equations," Communications in Nonlinear Science and Numerical Simulation, vol. 14, no. 2, pp. 443450, 2009.
[29] N. A. Kudryashov, "On "new travelling wave solutions" of the $\mathrm{KDV}$ and the KDV-BUrgers equations," Communications in Nonlinear Science and Numerical Simulation, vol. 14, no. 5, pp. 1891-1900, 2009.

[30] H. Demiray, "A note on the exact travelling wave solution to the KdV-Burgers equation," Wave Motion. An International Journal Reporting Research on Wave Phenomena, vol. 38, no. 4, pp. 367369, 2003.

[31] Z.-s. Feng and Q.-g. Meng, "Burgers-Korteweg-de Vries equation and its traveling solitary waves," Science China Mathematics, vol. 50, no. 3, pp. 412-422, 2007.

[32] Z. Feng and Y. Huang, "Approximate solution of the BurgersKorteweg-de Vries equation," Communications on Pure and Applied Analysis, vol. 6, no. 2, pp. 429-440, 2007.

[33] A. S. Abdel Rady, E. S. Osman, and M. Khalfallah, "The homogeneous balance method and its application to the BenjaminBONa-Mahoney (BBM) equation," Applied Mathematics and Computation, vol. 217, no. 4, pp. 1385-1390, 2010. 


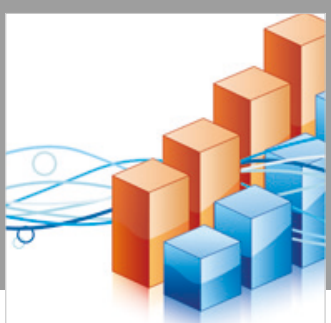

Advances in

Operations Research

\section{-n-m}
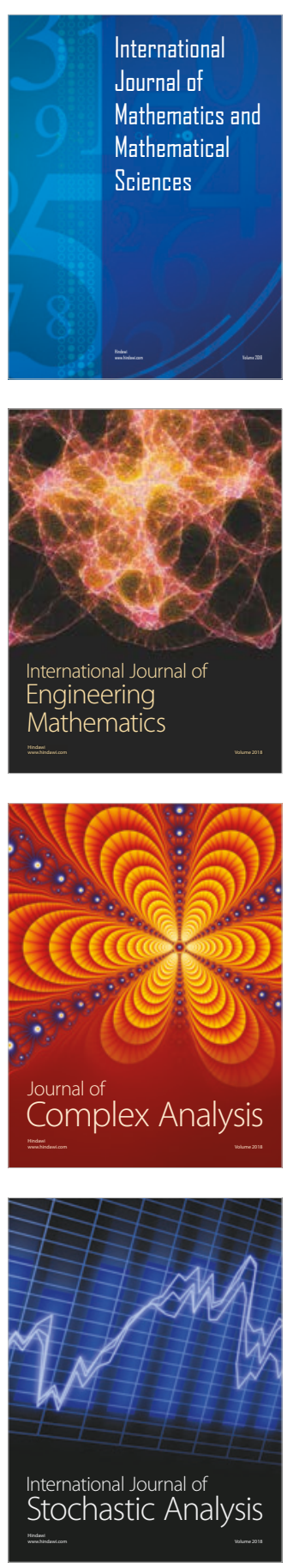
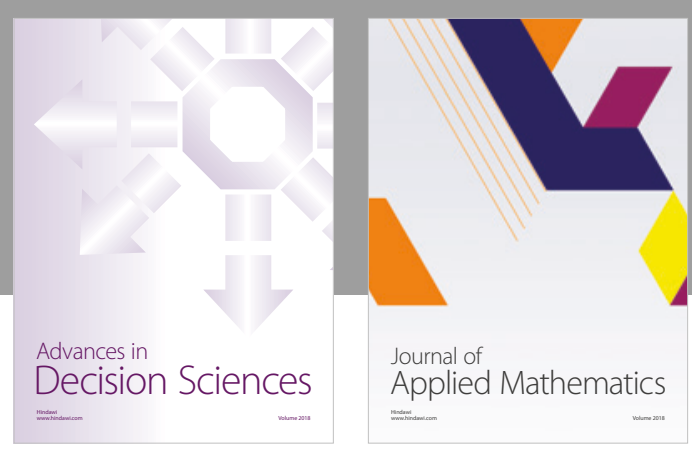

Journal of

Applied Mathematics
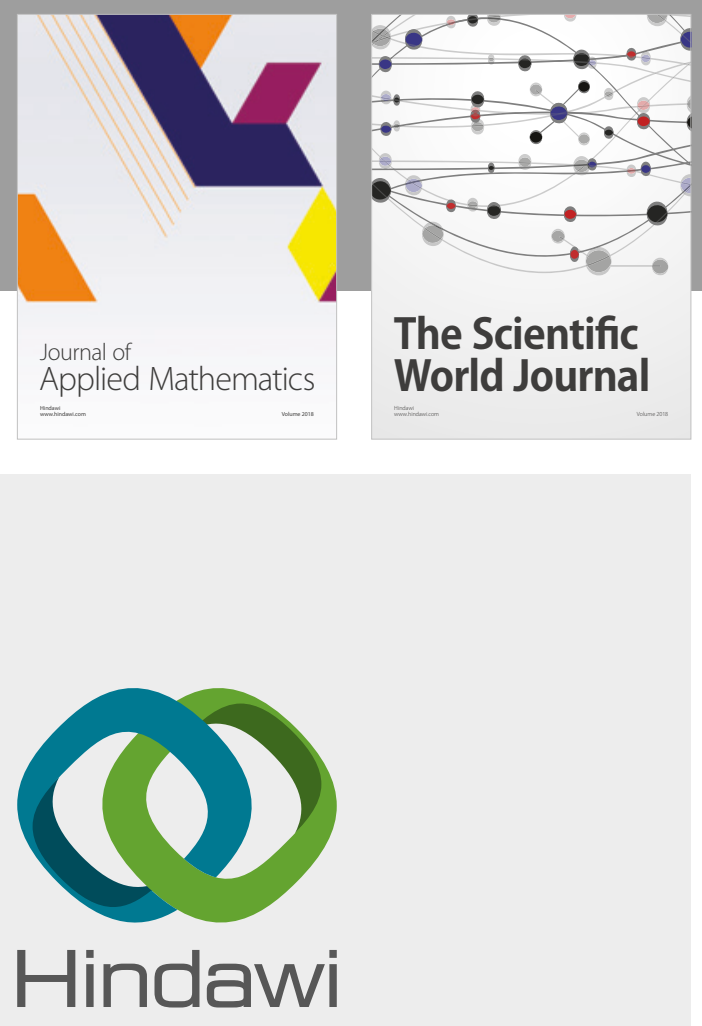

Submit your manuscripts at

www.hindawi.com

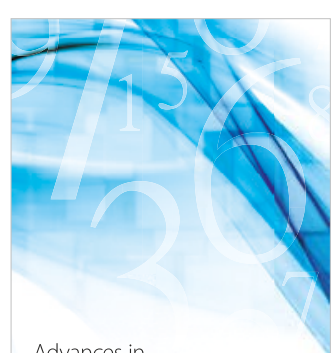

Advances in
Numerical Analysis
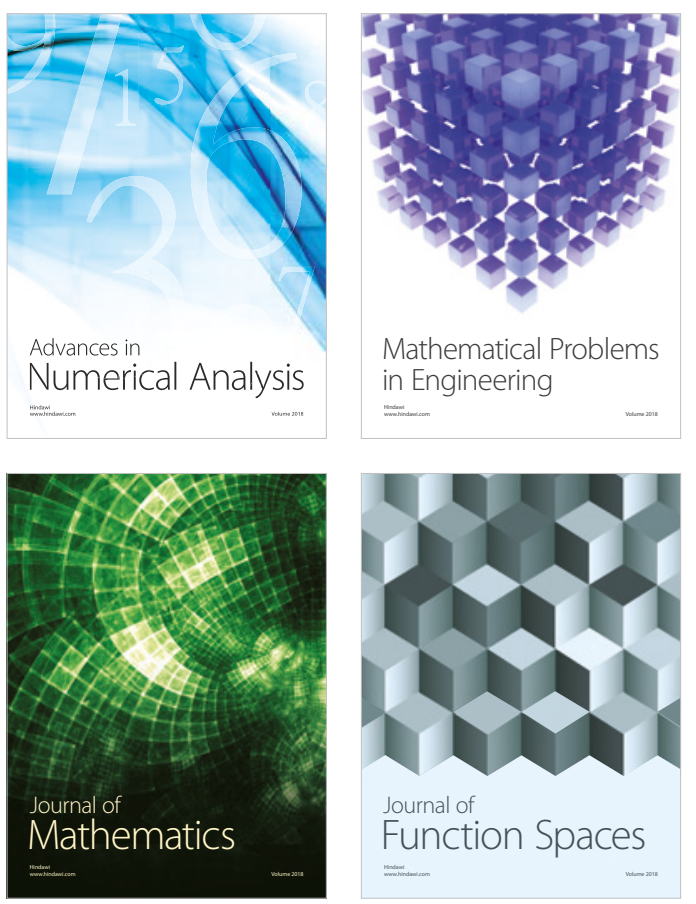

Mathematical Problems in Engineering

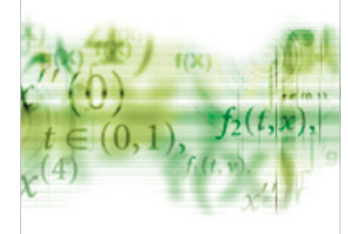

International Journal of

Differential Equations

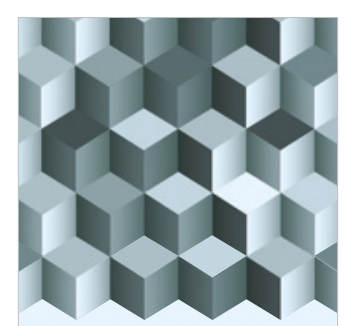

Journal of

Function Spaces

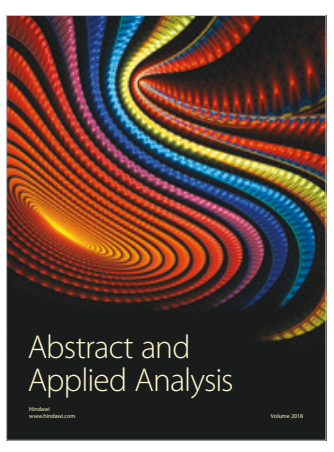

The Scientific

World Journal

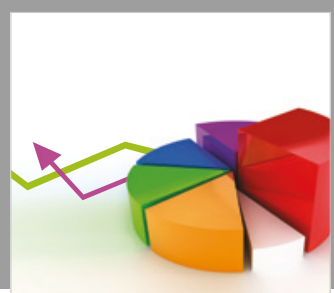

Journal of

Probability and Statistics
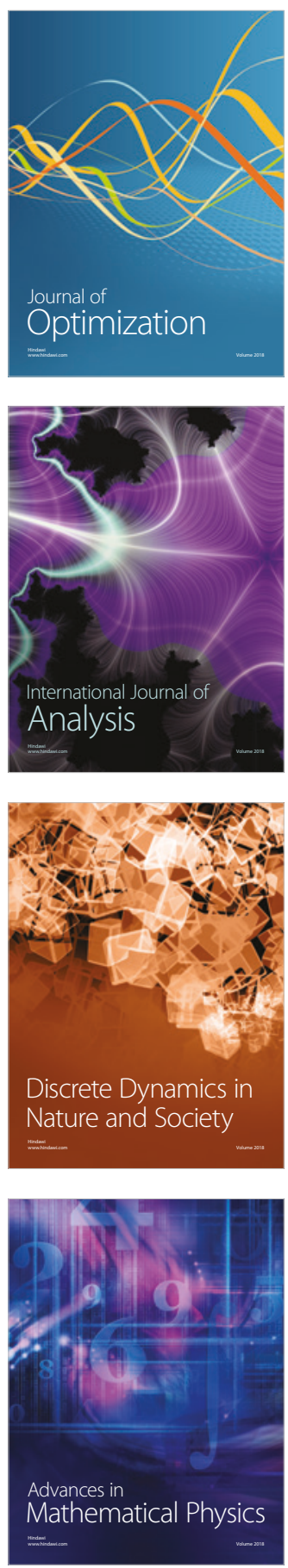